\title{
Feature Extraction Using Discrete Wavelet Transform for Gear Fault Diagnosis of Wind Turbine Gearbox
}

\author{
Rusmir Bajric, ${ }^{1}$ Ninoslav Zuber, ${ }^{2}$ Georgios Alexandros Skrimpas, ${ }^{3}$ and Nenad Mijatovic ${ }^{4}$ \\ ${ }^{1}$ EPC Elektroprivreda BiH, Kreka Coal Mines, 75000 Tuzla, Bosnia and Herzegovina \\ ${ }^{2}$ Faculty of Technical Sciences, University of Novi Sad, 21000 Novi Sad, Serbia \\ ${ }^{3}$ Bruel and Kjor Vibro, 2850 Nerum, Denmark \\ ${ }^{4}$ Technical University of Denmark, 2800 Lyngby, Denmark
}

Correspondence should be addressed to Ninoslav Zuber; zuber@uns.ac.rs

Received 1 July 2015; Revised 17 September 2015; Accepted 27 September 2015

Academic Editor: Chao Tao

Copyright (c) 2016 Rusmir Bajric et al. This is an open access article distributed under the Creative Commons Attribution License, which permits unrestricted use, distribution, and reproduction in any medium, provided the original work is properly cited.

\begin{abstract}
Vibration diagnosis is one of the most common techniques in condition evaluation of wind turbine equipped with gearbox. On the other side, gearbox is one of the key components of wind turbine drivetrain. Due to the stochastic operation of wind turbines, the gearbox shaft rotating speed changes with high percentage, which limits the application of traditional vibration signal processing techniques, such as fast Fourier transform. This paper investigates a new approach for wind turbine high speed shaft gear fault diagnosis using discrete wavelet transform and time synchronous averaging. First, the vibration signals are decomposed into a series of subbands signals with the use of a multiresolution analytical property of the discrete wavelet transform. Then, 22 condition indicators are extracted from the TSA signal, residual signal, and difference signal. Through the case study analysis, a new approach reveals the most relevant condition indicators based on vibrations that can be used for high speed shaft gear spalling fault diagnosis and their tracking abilities for fault degradation progression. It is also shown that the proposed approach enhances the gearbox fault diagnosis ability in wind turbines. The approach presented in this paper was programmed in Matlab environment using data acquired on a $2 \mathrm{MW}$ wind turbine.
\end{abstract}

\section{Introduction}

Wind energy is currently the fastest growing renewable energy source in the world. By the end of 2014, the global total installed wind capacity had reached more than $369 \mathrm{GW}$ with about $133 \mathrm{GW}$ of wind energy installed in Europe alone [1]. Wind turbines experience component failures which lead to increased operation and maintenance costs and, at the end, high cost of energy. At the same time, there is constant effort to reduce wind turbine downtime and increase availability. Wind turbine manufacturers have been exploring different drive train topologies ranging from multistage gearbox and induction generators to direct drive systems. Market share of direct drive turbines installations grew by $30 \%$ in 2014 and took $27 \%$ of the global market, according to World Wind Energy Market Update 2015, a slight decline in market share compared to 2013 despite good overall performance [2]. Direct drive turbines are still under development and they have some disadvantages, like large volume which leads to difficult transportation and manipulation.

In order to maintain the high availability of multistage gearbox wind turbine, condition monitoring of gearbox is essential. The major goal of wind turbine condition monitoring system (CMS) is to provide predictive, condition based maintenance that will improve safety, decrease maintenance costs, and increase system availability [3]. Most wind turbines are designed to operate for at least 20 years [4] and in addition to current market share of gearbox based wind turbines there is no doubt about the importance and necessity of CMS implementation. Despite technical challenges, vibration based CMS is the most popular diagnosis approach for wind turbine gearboxes [5]. Gearbox repair is complex and contributes to the long wind turbine downtime, especially when turbines are deployed offshore with requirement of special maintenance support vessel, crane ship, and good weather conditions for safe operation [6]. Standard method of gearbox 
fault diagnostics is data collection by accelerometers located on gearbox housing with the main purpose to collect vibration signals associated with transmission components, such as gears, shafts, and bearings. Operators of wind turbines pay attention to gearbox reliability due to the following reasons [6]: (1) the gearbox cost which is about $13 \%$ of overall wind turbine costs, (2) high cost of replacement and installation, (3) complex repair procedure, and (4) high revenue losses due to long downtime. For gearboxes operating at constant speed and load, traditional vibration based fault diagnostic techniques are reliable and allow end-users to accurately track fault progression over time. One of the approaches in gearbox fault diagnostics is direct feature extraction from the vibration signal. Features are used to reflect the condition of the gearbox components and in literature are referred to as condition indicator (CI). Features extracted from time domain signals usually reflect component degradation under constant operating conditions. However, reflection of component degradation will not be efficient under nonstationary operating conditions and will lead to fault misdiagnosis. Most of the frequency domain methods used in vibration based CMS today are based on the fast Fourier transform (FFT) techniques. Many vibration based fault diagnostic methods count on the fact that the speed and load are constant during data collection. Signals measured on the gearbox will change as load change while traditional FFT spectral methods cannot be used $[7,8]$. Furthermore, due to the stochastic operation of wind turbines, the running speed could change with high percentage which further limits the application of FFT based techniques. Hence, the vibration signals acquired on wind turbine gearbox are characterized as nonstationary [9] which leads to spectral smearing during application of FFT based techniques and creates uncertainty in the fault diagnosis. Večeř et al. [10] summarized a comprehensive selection of CIs for gears fault diagnosis and fault progression evaluation with some typical vibration signal analysis algorithms. Also, a detailed list of time domain and frequency domain features for gear fault detection and diagnosis is discussed in [11]. Other studies have been presented using National Renewable Energy Laboratory's Wind Turbine Gearbox Condition Monitoring Round Robin test rig with constant speed and load including GearMod algorithm [12], Sideband Index (SI) [3], sideband energy ratio (SER) [13], and sideband level (SBL) [14]. Wavelet transformation, which has the ability to provide information on both time and frequency domains, has been successfully applied in nonstationary vibration signal processing and fault diagnosis [15]. Recently, Siegel et al. [14] highlighted that the kurtosis based on residual signal is not the most appropriate technique for detection of high speed shaft (HSS) scuffing gear fault under stable operating condition of test rig wind turbine gearbox. However, this conclusion was reached using only 30 data sets obtained at three constant speeds and with $50 \%$ of rated power of the tested gearbox. The real data lifetime test (RDLT) behavior using this technique was not investigated. Also, residual signal contains rich fault information which is more sensitive to the gear faults, filtering out regular normal spectrum meshing frequencies and eliminating its disturbances [16]. In this paper, time synchronous averaging (TSA) signal is used as source signal for analysis due to their lower sensitivity to load variations, as suggested by Zhan et al. [17]. The HSS is found to be the most unreliable gearbox stage [18]. A recent effort in wind turbine condition monitoring industry has focused to automate the data interpretation and improve the accuracy and reliability of diagnosis decision. From the earlier studies, it seems there is a need for more in-depth evaluation of the various vibration based gear CIs on the RDLT wind turbine gearbox. Although various CIs provided successful results under experimental settings, their ability in RDLT was not sufficiently studied. There are many reasons to conduct gearbox condition monitoring research within the wind turbine. The idea of this paper was triggered by a real multimegawatt wind turbine gearbox failure, where the HSS gear was severely damaged by spalling fault on single tooth. At the end, this fault resulted in wind turbine stop. This study aims to contribute the area of wind turbine vibration based condition monitoring by providing a detailed investigation of selected 22 CIs for HSS gear fault diagnosis and to estimate the properties of selected CIs to track gear fault progression. The study is conducted on the RDLT wind turbine gearbox, thus providing a more compelling investigation of whether the CIs are applicable to a CMS. A new approach which uses the discrete wavelet transform (DWT) to filter out TSA signals and its special transform "residual and difference signal" in process of gear faults CIs extraction is presented and evaluated in this paper. The proposed approach was realized with Matlab coding and validated with RDLT wind turbine data.

The remainder of the paper is organized as follows. In Section 2, we provide a quick overview of the discrete wavelet transformation and employment of residual and difference signal and CIs used in the study. In Section 3, wind turbine gearbox experiment and experimental procedure are described. Fault diagnosis approach which is presented in Section 3 is analyzed in Section 4, and the effect of selected approach is discussed. The conclusion is given in Section 5.

\section{Vibration Signal Processing and Feature Extraction}

2.1. Wavelet Transform. Depending on wind speed, wind turbines operate in time varying conditions that make fault diagnosis complicated. In this context, the classical application of Fourier based spectrum methods for processing the time varying signals does not give reliable results. Application of wavelet transformation in signal processing for fault diagnosis is a well-known technique which overcomes the problems that other signal processing techniques have. Unlike spectral analysis that represents a signal as sum of sinusoidal functions, wavelet transform decomposes the signal into wavelets of various scales in time-domain with variable window sizes and revealing the local structure in time frequency domain. Wavelet transform, which is capable of providing both time and frequency domain information simultaneously has been successfully used in nonstationary vibration signal processing and fault diagnosis [7]. The advantages of wavelet transform [19] are as follows: (1) it 
is more effective for transient features extraction and (2) it extracts signal features over the entire spectrum, without requiring a dominant frequency band.

The wavelet transformation uses the basic wavelet functions, also referred to as mother wavelets, which can dilatecompress and translate domain based on two parameters: scale-frequency and translation-time shift in order to apply short windows at low scales-high frequencies and long windows at high scales-low frequencies. The latter provides greater resolution in time for high frequency components of a signal and greater resolution in frequency for low frequency components.

2.2. Gear Fault Feature Extraction Using DWT. Fast Fourier transform is still the most used signal processing method for gearbox fault diagnosis that can be found in commercially available online monitoring systems. On the other side, timefrequency analyses methods have the ability to display both time information and frequency information of a signal. The multiresolution analysis ability of DWT makes it suitable for revealing fault related information from nonstationary signals acquired on rotating machineries [20]. Frequency bandwidth of the vibration spectrum for gearbox fault diagnosis needs to be wide in order to cover higher harmonics of fundamental meshing frequency and also needs to have high frequency resolution in order to extract information around fundamental meshing frequency and its harmonics. The basic analysis wavelet $\psi(t)$ is a square integrable function, and it meets the following relationship [21]:

$$
C_{\psi}=\int_{R} \frac{|\Psi(\omega)|^{2}}{|\omega|} d \omega<\infty,
$$

where $\Psi(\omega)$ is the Fourier transform of $\psi(t)$. Through translation and dilatation, a member of the function can be derived from the $\psi(t)$. The equation can be described as follows:

$$
\psi_{a, b}(t)=|a|^{-1 / 2} \psi\left(\frac{t-b}{a}\right),
$$

where $\psi_{a, b}(t)$ is a member of wavelet mother function and $a$ and $b$ represent the scale parameter and translation parameter, respectively. As seen in (2) and (3), $\psi_{a, b}(t)$ can be regarded as a window function. $a$ and $b$ are used to adjust the frequency and time location of the wavelet. Small $a$ offers high-frequency resolution and is useful in extracting high-frequency components of signals. Small $a$ increases in response to the frequency resolution decrease but increase in time resolution and therefore low-frequency components are much easier to extract. DWT is derived through the discretization of parameters $a$ and $b$. Generally, $a$ is replaced by $2 j$ and $b$ is replaced by $k 2 j(j, k \in Z)$ and can be shown as

$$
W_{\psi}(j, k)=\int x(t) \psi_{j, k}(t) d t
$$

where $\psi_{j, k}(t)=2^{-j / 2} \psi\left(2^{-j} t-k\right)$ and $x(t)$ is finite-energy signal. The Mallat algorithm [21, 22] implements DWT using pyramidal structure where time dilation is accomplished by

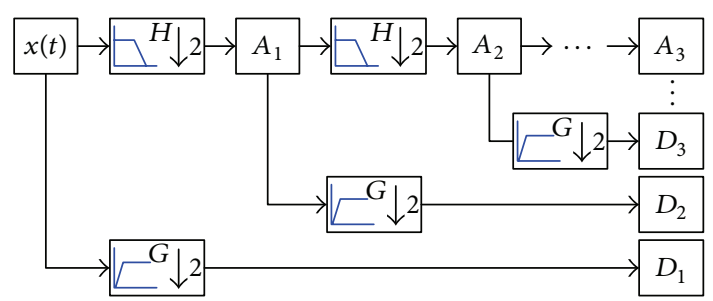

Figure 1: Decomposition procedure of $J$-level DWT.

downsampling for every stage of wavelet decomposition. Wavelet filters are used for decomposition and reconstruction. It is shown in

$$
\begin{aligned}
& A_{0}[x(t)]=x(t), \\
& A_{j}[x(t)]=\sum_{k} H(2 t-k) A_{j-1}[x(t)], \\
& D_{j}[x(t)]=\sum_{k} G(2 t-k) A_{j-1}[x(t)],
\end{aligned}
$$

where $x(t)$ is the original signal and $j$ is the decomposition level $(j=1,2, \ldots, J) . H$ and $G$ are wavelet decomposition filters for low-pass filtering and high pass filtering, respectively. $A_{j}$ and $D_{j}$ are the low frequency wavelet coefficients (approximations) and the high frequency wavelet coefficients (details) of signal $x(t)$ at the $j$ th level, respectively. The decomposition procedure of a $J$-level DWT is shown in Figure 1. It can be seen that $D_{j}$ and $A_{j}$ are obtained through high-pass filtering and low pass filtering with downsampling at each level. After the signal $x(t)$ is decomposed by the $J$-level DWT, $D_{j}$ at each level and $A_{j}$ at the $J$ th level are obtained. Therefore, the DWT based on Mallat algorithm can be represented as a filtering process where the signal is decomposed into a series of subbands.

More detailed analytical bases of the wavelet technique can be found in [22]. DWT, based on subband coding, is known as a fast computation wavelet transform that exploits the relationship between the coefficients at adjacent scales. Such implementation reduces the computational time which renders it much more suitable for online fault diagnosis. With regard to the type of mother wavelet, a 5th order of Daubechies family $d b 5$ was chosen, although other families (Symlet) also allow a clear detection of the gear faults [23]. Actually, a high order of mother wavelet is recommended for minimizing the overlapping effect at the expense of a higher computation time [23]. However, thanks to the robustness of the used method, the use of lower order mother wavelet is able to provide satisfactory results.

2.3. Time Synchronous Averaging. As mentioned, regular spectrum based methods are not reliable for wind turbine gearbox fault diagnosis. The majority of the energy in the healthy state of the gear is concentrated at the fundamental meshing frequency and its harmonic. If sampling of vibration signal is synchronized with the rotation of a selected shaft over many revolutions the result will be a signal called TSA signal [24], which contains only signal components which 


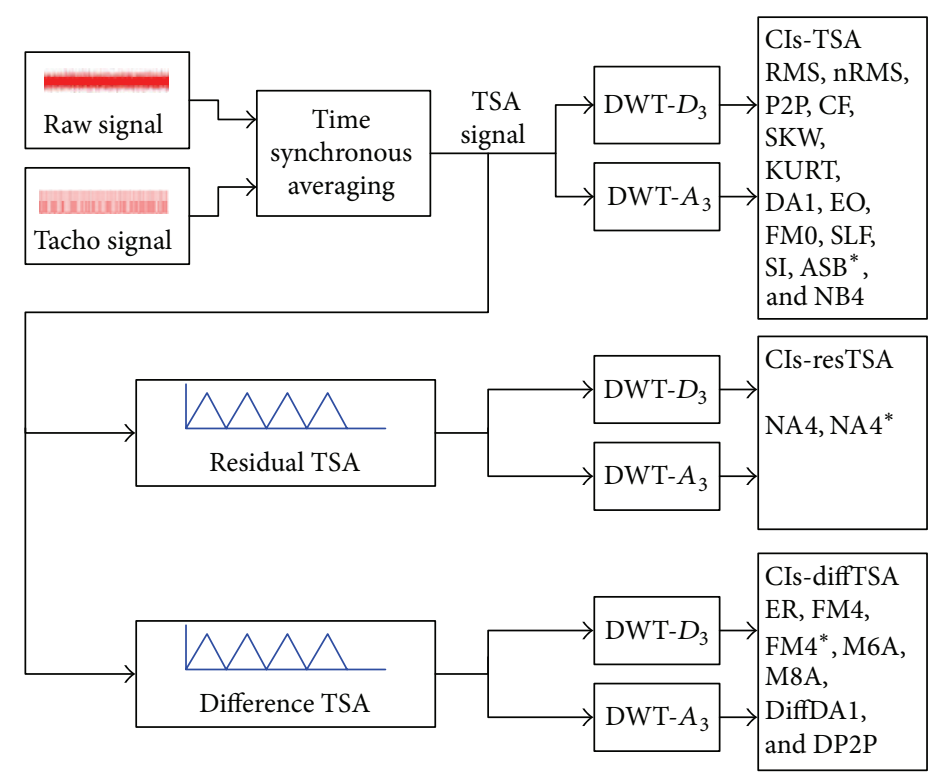

FIGURE 2: Processing flow chart for feature extraction methods for vibration analysis.

are synchronous with selected shaft. TSA is a well-known and efficient algorithm for fault diagnosis of gears. It has the ability to extract deterministic signals and enhance synchronous vibration related components, while suppressing nonsynchronous components, electrical noise, bearing vibrations in particular, and vibrations related to other gearbox shafts or nearby machinery. The quality of TSA signal highly depends on the number of averages in the TSA process. However, alhthough TSA is a very powerful algorithm, one of the potential problems with TSA implementation in commercial wind turbine vibration based CMS is the necessity for reference sensor on all gearbox stages. There are different definitions of "residual signal" but the major goal in generating it is to remove the regular meshing pattern from the time domain signal. Gear residual signal is special transformation of TSA vibration signal from gears in mesh and usually shows evidence of presence of faults earlier and more clearly than TSA signal. It is also less sensitive to the varying load than TSA signal [7]. It is common to remove the first five shaft harmonics, gear mesh frequency, and all gear mesh harmonics frequency from the residual signal [14]. The first-order sidebands needs to be removed from the "residual signals" in order to obtain "difference signal".

2.4. Extraction of Features. The most important vibration spectral components for gear fault diagnosis are gear mesh frequencies, their harmonics and sidebands generated by modulation due to geometric errors of tooth profile, meshing errors, and gear distribute/localized fault. Sideband amplitudes around gear mesh frequencies remain constant over the time for gear in good condition. Therefore, the number and the amplitude of sideband could be used to indicate presence of fault in meshing gears [25]. To enable determination of gear condition, to track its degradation progression, and to extract characteristic features, many vibration based
TABLE 1: Signals of interest and used CIs.

\begin{tabular}{lll}
\hline Signals type & Name of signal & Used CIs \\
\hline \multirow{2}{*}{ TSA } & & RMS, nRMS, P2P, CF, \\
& TSA & SKW, KURT, DA1, EO, \\
& & FM0, SLF, SI, ASB ${ }^{*}$, and \\
& & NB4 \\
\hline Residual TSA & resTSA & NA4, NA4* \\
\hline \multirow{2}{*}{ Difference TSA } & \multirow{2}{*}{ diffTSA } & ER, FM4, FM4* ${ }^{*}$, M6A, \\
& & M8A, DiffDA1, and \\
& & DiffP2P \\
\hline
\end{tabular}

diagnostic approaches have been developed. In literature, those features are known as CIs. The ability to diagnose gear faults while minimizing the probability of false alarms is the primary goal during CI selection [3]. Most of gear CIs are based on TSA signal and the first step in calculating these CIs is to obtain the TSA signal. Number of CIs could be used for gear fault diagnosis based on signal processing techniques and kinematics of machine. An extensive discussion of the gearbox vibration CIs analysis can be found in $[10,26]$. Many indicators are then applied to the TSA signal, residual signal, and difference signal. An overview of the signals used to extract CIs is provided in Table 1. Figure 2 shows the CIs used in this paper, associated with the different signal processing techniques.

The following 22 CIs were extracted: root mean square (RMS) and normalized RMS (nRMS) [27] whichis defined as RMS of a vibration acceleration signal with amplitude normalized by signal's peak value:

$$
\mathrm{nRMS}=1-\frac{\operatorname{RMS}(x)}{|\max (x)|} .
$$

Peak to Peak (P2P), Crest Factor (CF), Skewness (SKW), Kurtosis (KURT) are well known CIs, and Data Algorithm 
One (DA1) [28] is expressed by (6) and is defined as RMS of the TSA signal subtracted from the mean of the TSA:

$$
\mathrm{DA} 1=\operatorname{RMS}(\mathrm{TSA}-\overline{\mathrm{TSA}}) .
$$

Energy Operator (EO) [10] is defined as the normalized kurtosis from the signal where each point is computed as the difference of two squared neighborhood points of the original signal. Zero-order figure of merit (FM0) [10] is defined as the peak to peak level of the signal divided by the sum of amplitude at the GMF and its corresponding harmonics. Sideband Level Factor (SLF) [28] is expressed by (7) defined as sum of the first-order sideband amplitudes of the fundamental gear meshing frequency normalized by the RMS of the synchronous time average signal:

$$
\text { SLF }=\frac{\sum \text { magnitude of first order side bands }}{\operatorname{RMS}(x)} .
$$

NB4 defined in [29], Sideband Index (SI) [28] is expressed by (8) defined as the average of the first-order sidebands of the fundamental gear meshing frequency:

$$
\mathrm{SI}=\frac{\sum \text { magnitude of first order side bands }}{2},
$$

and amplitude of sidebands $\left(\mathrm{ASB}^{*}\right)$ [27] is defined as relationship of amplitude of sidebands (ASB) and the amplitudes of the GMF and its harmonics (AGMF)

$$
\mathrm{ASB}^{*}=\frac{\mathrm{ASB}}{\mathrm{AGMF}}
$$

are applied to TSA signal.

NA4 [30] evaluated by (10) is defined as the kurtosis of a signal normalized by square average variance for the run ensemble

$$
\mathrm{NA} 4=\frac{N \sum_{i=1}^{N}\left(r_{i}-\bar{r}_{i}\right)^{4}}{\left[\sum_{i=1}^{N}\left(r_{i}-\bar{r}_{i}\right)^{2}\right]^{2}} .
$$

$\mathrm{NA}^{*}{ }^{*}[30]$ is defined as the kurtosis of a signal normalized by square average variance for the gearbox in good condition. This CI is obtained using the "residual signal". Finally the FM4, FM4* ${ }^{*}$ Energy Ratio (ER) [10], M6A, and M8A [31] are applied to the difference signal estimated by removing firstorder sidebands from the residual signals. FM4 is given by (11) and is defined as the kurtosis of a difference signal:

$$
\mathrm{FM} 4=\frac{N \sum_{i=1}^{N}\left(d_{i}-\bar{d}_{i}\right)^{4}}{\left[\sum_{i=1}^{N}\left(d_{i}-\bar{d}_{i}\right)^{2}\right]^{2}} .
$$

The ER given by (12) is defined as the ratio of the RMS of the difference signal to the RMS of the signal containing only the regular meshing components (TSA signal):

$$
\mathrm{ER}=\frac{\mathrm{RMS}_{d}}{\mathrm{RMS}_{\mathrm{TSA}}} .
$$

The M6A given by (13) is

$$
\mathrm{M} 6 \mathrm{~A}=\frac{N^{2} \sum_{i=1}^{N}\left(d_{i}-\bar{d}_{i}\right)^{6}}{\left[\sum_{i=1}^{N}\left(d_{i}-\bar{d}_{i}\right)^{2}\right]^{3}},
$$

and the M8A given by (14) is

$$
\text { M8A }=\frac{N^{3} \sum_{i=1}^{N}\left(d_{i}-\bar{d}_{i}\right)^{8}}{\left[\sum_{i=1}^{N}\left(d_{i}-\bar{d}_{i}\right)^{2}\right]^{4}},
$$

which generally are more sensitive to peaks in the signal as a consequence of the use of high-order statistics. Recently, two gear CIs in health and usage monitoring systems (HUMS), DA1 of the difference signal (DiffDA1), and peak to peak of the difference signal (DiffP2P) have been investigated [26]. After resampling the signal from the HSS accelerometer, the TSA signal is computed taking into account the tachometer signal also from the HSS shaft as reference. In our case, a filtering process is applied to the TSA signal which involves removing the fundamental gear mesh frequency (GMF) and its first five harmonics to generate what is called the residual signal (resTSA). A filtering process is applied that involves removing the first order sidebands from the residual signals in order to obtain difference signal (diffTSA). Finally, the TSA, the resTSA, and the diffTSA signals are then filtered by the DWT using the approximation and detail coefficients, from which a number of CIs is extracted. Time waveform of vibration signal will deform during gear meshing processes if there is a fault on gear and this is usually manifested with increase amplitudes of gear meshing frequency and its harmonics. Gear mesh related amplitudes are usually very small if there is no fault at meshing gears. There is more energy at mesh frequency and its harmonics while fault progress and fault progress could be supervised as an increment in higher harmonics of mesh frequency. This is the reason why it is common to analyze at least the first three harmonics of the gear meshing frequency. As reported in Table 2, the characteristic fault frequencies of the HSS gear can be observed, respectively, on approximation $A_{3}$ and detail $D_{3}$ coefficients. Hence, the DWT was done for only 3 decomposition levels.

Approximation $A_{3}$ and details coefficients $D_{3}$ are used covering first four GMFs (1xGMF-728 Hz, 2xGMF-1456 Hz, $3 x G M F-2184 \mathrm{~Hz}$, and $4 x G M F-2912 \mathrm{~Hz}$ ) while others are omitted from analysis. Finally, these signals were analyzed by the proposed approach using the Matlab code developed by the authors.

\section{Wind Turbine Gearbox Fault Diagnosis}

3.1. Wind Turbine Description and Data Acquisition. In order to extract the appropriate condition indicators and evaluate their historical trends, we present in this section the wind turbine gearbox experiment and advantages of using TSA and residual and different signal. To evaluate the proposed approach, wind turbine gearbox RDLT vibration data history is used collected by a vibration data acquisition system. 
TABLE 2: DWT 3 level frequency bands.

\begin{tabular}{lccc}
\hline $\begin{array}{l}\text { Approximations } \\
\text { " } A_{j} \text { " }\end{array}$ & $\begin{array}{c}\text { Frequency band } \\
(\mathrm{kHz})\end{array}$ & $\begin{array}{c}\text { Details } \\
\text { " } D_{j} "\end{array}$ & $\begin{array}{c}\text { Frequency } \\
\text { band }(\mathrm{kHz})\end{array}$ \\
\hline$A_{3}$ & $(0-1.6)$ & $D_{3}$ & $(1.6-3.2)$ \\
$A_{2}$ & $(0-3.2)$ & $D_{2}$ & $(3.2-6.4)$ \\
$A_{1}$ & $(0-6.4)$ & $D_{1}$ & $(6.4-12.8)$ \\
\hline
\end{tabular}

The vibration signals were acquired by accelerometers mounted on the gearbox housing close to HSS shaft supporting bearing. The rotational speed of the HSS shaft was obtained by tachometer. Moreover, the vibration and tachometer signals were sampled at $25.6 \mathrm{kHz}$ with 10.24 seconds lengths where 25 sets of vibration and tachometers signals were analyzed over 4 months.

Furthermore, in order to analyze data under similar wind turbine load, data sets are classified. Only data sets from $0.7 \mathrm{MW}$ to $1.4 \mathrm{MW}$ are used while others were neglected. Gear meshing frequency (GMF) of HSS pinion and gear was $728 \mathrm{~Hz}$ with $28 \mathrm{~Hz}$ HSS shaft running speed. Wind turbine gearbox ratio is close to $1: 110$ rated at $2.0 \mathrm{MW}$ nominal electric power. The gearbox is a three-stage unit, with the first stage being planetary and the remaining two stages being parallel gears. Wind turbine gearbox used for evaluation of selected CIs is three-stage design gearbox, composed of one planetary stage and two parallel shaft stages.

This design type is widely used configuration for wind turbine drivetrains, as shown in Figure 3, and illustrates the internal configuration of the wind turbine gearbox under evaluation. The low speed shaft (LSS) stage-planetary stage includes the planetary gears in a planet carrier coaxial with a sun gear and a ring gear. Planet carrier is connected to main blade rotor and transfer motion to sun gear via the planet gears. The intermediate speed shaft (ISS) stage uses parallel helical gears as does the high speed shaft (HSS), which is then coupled to generator drive end. Internal configuration of the wind turbine gearbox is given in [3]. The gear fault under evaluation in this study was HSS gear single tooth severe spalling. The HSS running speed has a profile as shown in Figure 4 for data set acquired on 05/08/2014.

For specific span, there was approximately a $1.2 \mathrm{~Hz}$ HSS speed change. Even though the HSS shaft speed variation seems relatively small in this case, this kind of variation is large enough to create smearing in the frequency based spectrum analysis, especially at high orders of the HSS rotational frequency, such as the HSS gear meshing frequency and its harmonics.

\section{Diagnosis Results and Discussion}

Before the CIs are extracted, it is important to evaluate the performance of TSA and the resTSA to observe how the vibration signals change for undamaged and damaged HSS gear. TSA is first performed on the signal over 250 averages, taking the HSS shaft rotation as reference. The effectiveness of this technique in case with damaged HSS gear is shown in Figure 5 using vibration signal power spectrum.

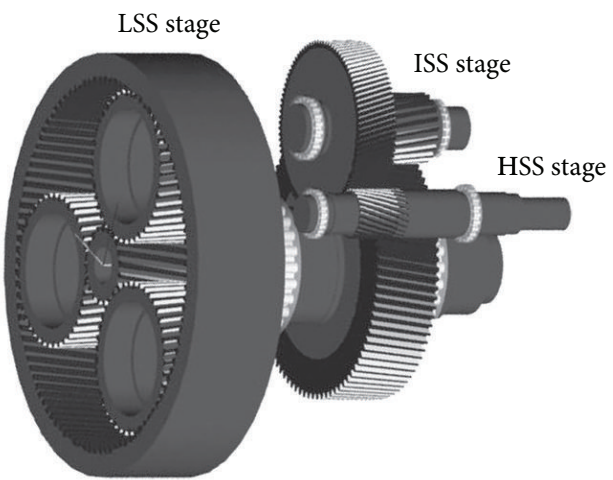

FIGURE 3: Wind turbine gearbox with one planetary and two parallel stages, adapted from [6].

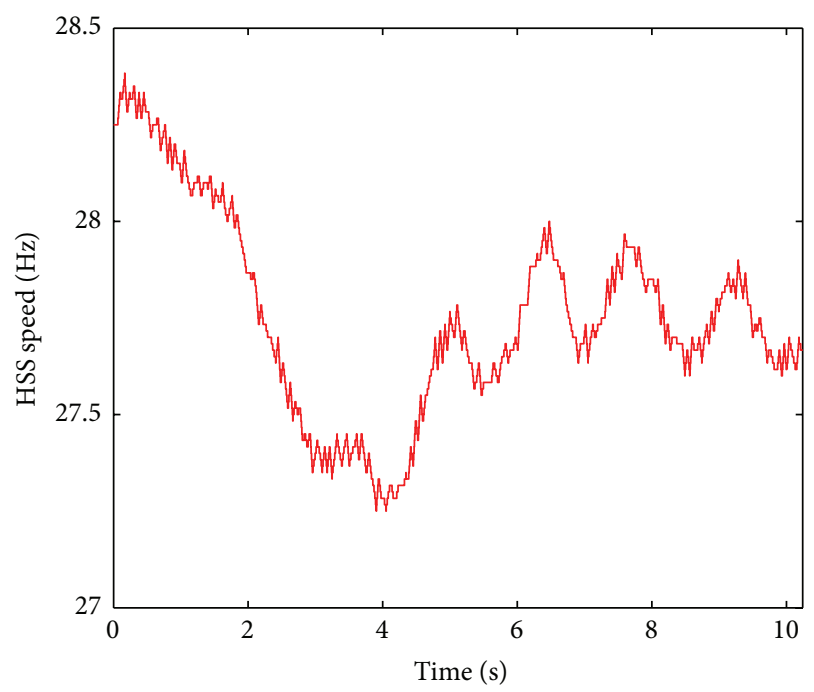

FIGURE 4: HSS speed profile.

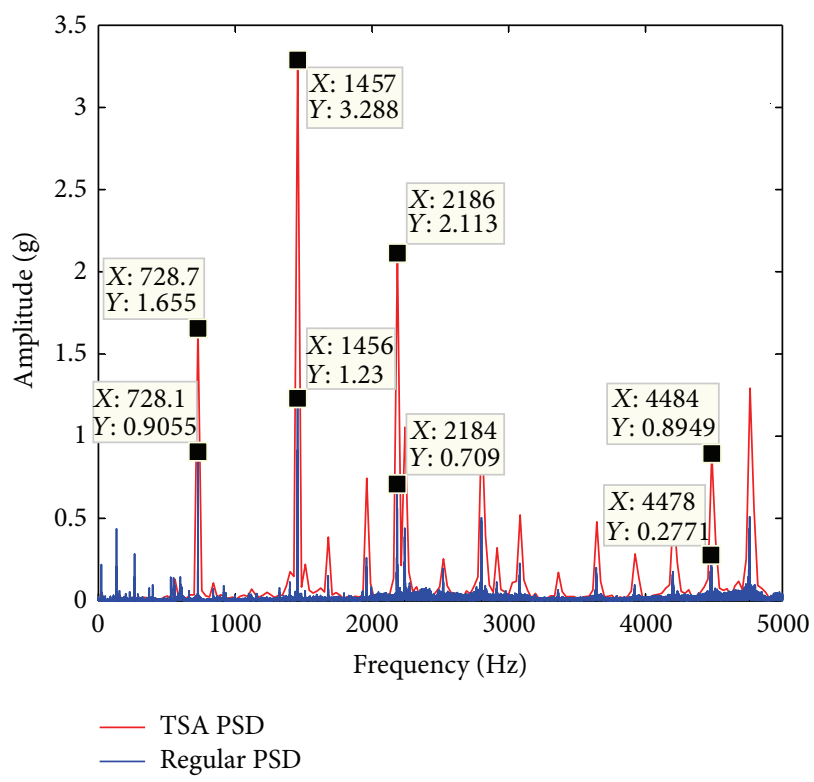

FIGURE 5: Power spectrum comparison between the regular signal and TSA signal for damaged HSS gear. 


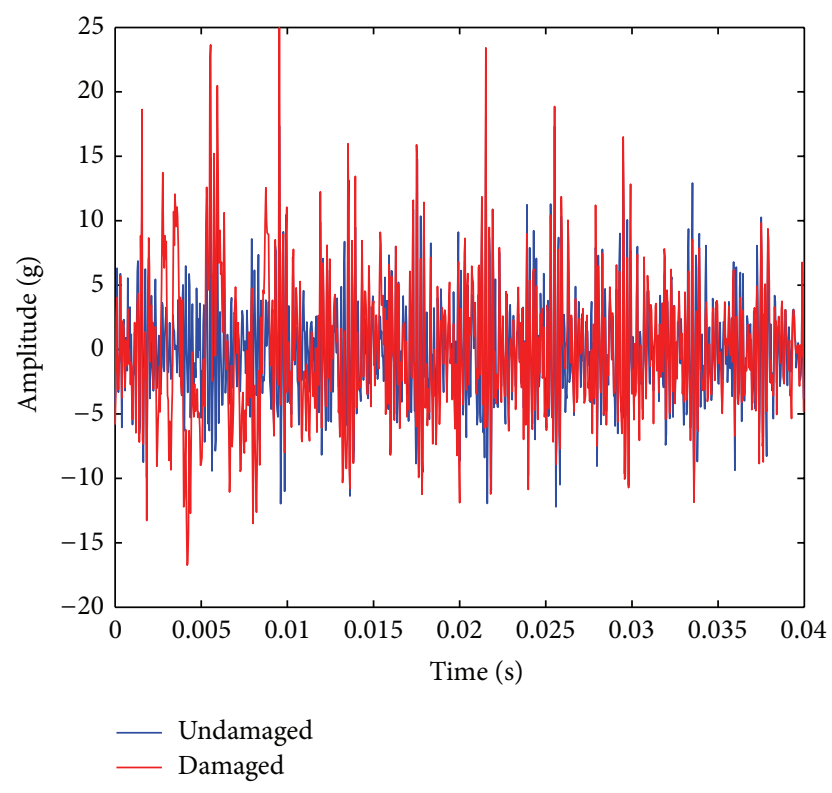

FIGURE 6: Residual signal of HSS gear (one complete revolution of HSS gear).

It shows that the asynchronous components as well as the noise floor are significantly reduced, while frequencies that are synchronous with the rotation of the HSS gear have emerged in the spectrum; that is, the GMF and its harmonics are clearly visible indicating gear meshing irregularities and fault presence.

Comparing regular and TSA power spectra at the high speed GMFs and high-order harmonics, the amplitude difference becomes significant, especially at the higher orders GMFs. Although the full frequency range spectrum is available, for clarity, only the first $5 \mathrm{kHz}$ frequency range is shown in Figure 5. In order to estimate the residual signals, infinite impulse response notch filters are used. This type of filter attenuates frequencies in a narrow stop band around a cut-off frequency. Typical results for the residual signal are presented in Figure 6 for datasets of undamaged and damaged HSS gear.

4.1. Extraction of Features. To evaluate the diagnosis capabilities in the case of HSS gear spalling fault, features were implemented and processed for wind turbine gearbox RDLT. Generally, features can be divided into two groups, statistical condition indicators and power condition indicators as follows:

(1) Statistical CIs offer an overview on the trend of signals and therefore on the operation condition of the machine. CIs RMS, nRMS, P2P, CF, SKW, KURT, DA1, EO, FM0, SLF, SI, ASB ${ }^{*}$, and NB4 are extracted from the TSA signal. NA4 and NA4* are extracted from residual signal where ER, FM4, FM4*, M6A, M8A, DiffDA1, and DiffP2P are extracted from difference signal.

(2) Power in bands CIs or frequency domain based CIs are signature typical use as second approach prior to

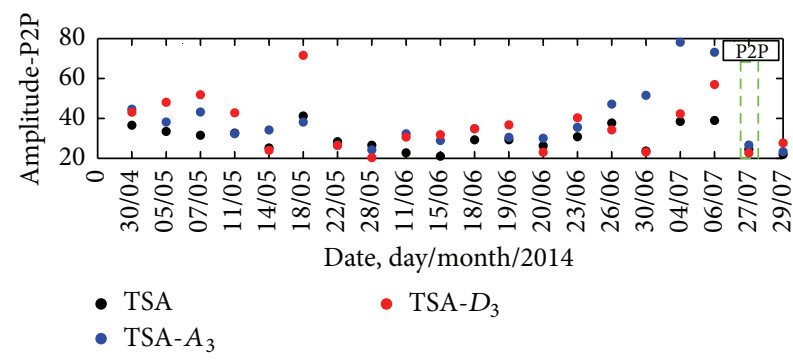

FIGURE 7: P2P over RDLT.

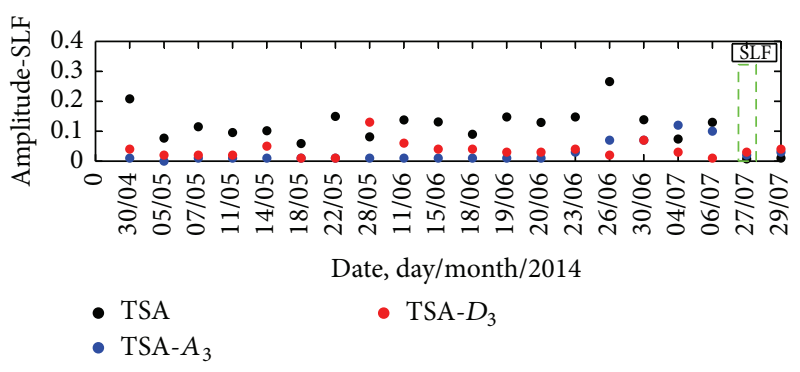

FIGURE 8: Sideband Level Factor over RDLT.

applying more advanced processing methods. These CIs allow tracking the changes in energy around some frequencies related to defects in gear components to be monitored. These CIs are extracted from TSA signal and have been designed for fault detection, based on the analysis presented earlier in this paper. FM0, SLF, SI, and $\mathrm{ASB}^{*}$ are power in bands CIs used in this study.

The historical trends of all the CIs defined in the earlier section are depicted in Figures 7-12. Both the clarity and the fluctuation level of the historical trends for the CIs are considered as criteria for selecting relevant features from the generated parameters used for HSS gear fault diagnosis and degradation progression over the wind turbine RDLT gearbox. A new wind turbine gearbox was installed on $27 / 07 / 2014$ and is marked on figures with green rectangle.

The results illustrate that the statistical condition indicators RMS, nRMS, CF, SKW, KURT, DAl, EO, FM0, ASB ${ }^{*}$, and NB4 based on the TSA signal do not reveal a linear increase over the wind turbine RDLT. Only P2P as statistical CI shows a logical increase over the wind turbine RDLT as shown in Figure 7.

From 18/06/2014 this indicator had linear increase, which may reflect the beginning of the deterioration of the HSS gear. The starting point of the fault on HSS gear is also confirmed by SLF and SI as power in band CIs which significantly increases from 23/06/2014, as shown in Figures 8 and 9, respectively.

We also remark that the SLF and SI indicators are more pertinent when extracted from the approximation coefficient $A_{3}$ (low frequency) signal than the indicators extracted from the regular TSA signal and detail coefficient $D_{3}$ (high frequency) signal. In addition, P2P, SLF, SI, and NA4* extracted from approximation coefficient $A_{3}$ reflect real fault progress 


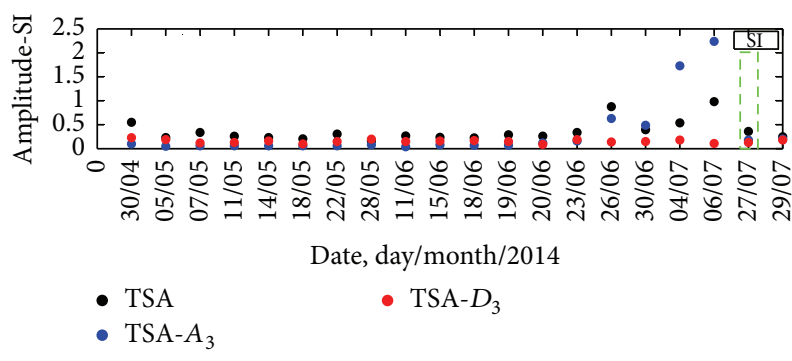

FIgURE 9: Sideband Index over RDLT.

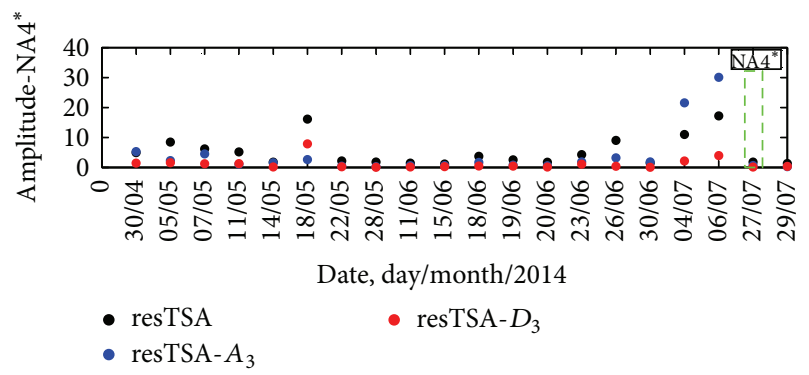

FIGURE 10: NA4* over RDLT.

compered to regular TSA signal. Those two CIs are associated with gear mesh frequencies and sidebands calculated from the frequency domain and SI as condition indicator is more robust than others' power in band CIs in tracking HSS gear fault progression over the time.

We also remark that all other analyzed statistical and power in band CIs based on TSA signal do not reveal a linear increase over the wind turbine RDLT (fault progression).

The NA4 CIs extracted from residual signal do not reveal a linear increase over the wind turbine RDLT. In general, NA4 condition indicator was developed as one that is more sensitive to progressing damage. By normalizing the squared variance for gear in gsood condition NA4* CI becomes more robust when progressive damage occurs. The study results illustrate that this is the case for wind turbine single tooth spalling HSS gear fault with rapid increase from 26/06/2014 as shown in Figure 10. This indicator is more pertinent when extracted from the approximation coefficient $A_{3}$ (low frequency) signal than the indicators extracted from the regular TSA signal and detail coefficient $D_{3}$ (high frequency) signal. What is more, this indicator very efficiently reveals when the new gearbox was installed.

In general, CIs extracted form difference signal are designed to reveal change of GMF high-order sidebands. ER does not reveal a linear increase over the wind turbine RDLT and even increase after the replacement of gearbox. This is expected because ER is known as condition indicator tracking heavy wear, where more than one tooth on the gear is damaged. FM4 have shown similar behavior with higher rate of fluctuation. FM4* difference signal based CI reveals a linear increase over the wind turbine RDLT and this indicator is capable of revealing when the new gearbox was installed as shown in Figure 11. This indicator is more

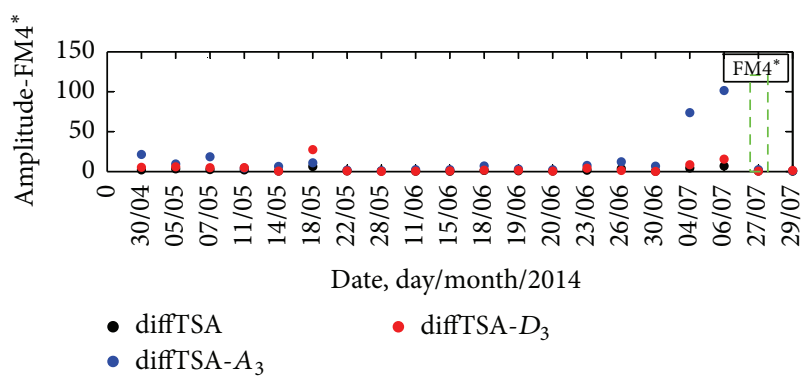

FIGURE 11: FM4* over RDLT.

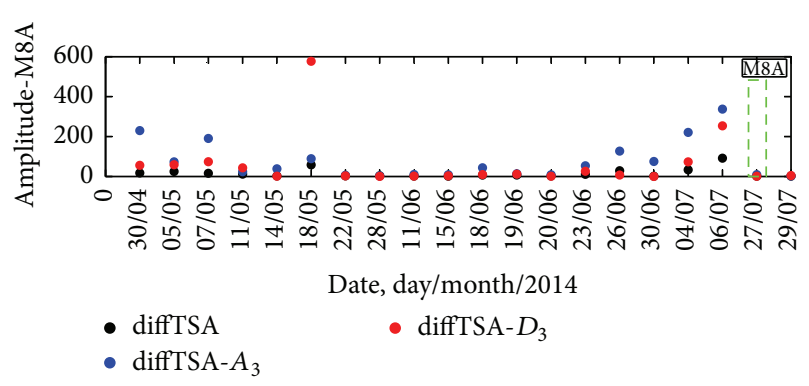

FIgURE 12: M8A over RDLT.

pertinent when extracted from the approximation coefficient $A_{3}$ (low frequency) signal than the indicators extracted from the regular TSA signal and detail coefficient $D_{3}$ (high frequency) signal. M8A difference signal based CI reveals a linear increase over the duration of RDLT and has good correlation with the time of gearbox replacement as shown in Figure 12. We also noticed that this indicator is more pertinent when extracted from the approximation coefficient $A_{3}$ (low frequency) signal than the indicators extracted from the regular TSA signal and detail coefficient $D_{3}$ (high frequency) signal. This condition indicator is designed to detect surface damage on machinery components which is the case with HSS gear fault, in this case spalling. Other CIs based on difference signal do not reveal linear increase over the wind turbine RDLT and some of them even increase after the replacement of gearbox with high level of fluctuation. The statistical condition indicators M6A, DiffDA1, and DiffP2P based on the difference signal do not reveal a linear increase over the wind turbine RDLT.

\section{Conclusion}

One of the most important insights to be drawn from this work is choosing a suitable reference for TSA and CI that can lead to earlier diagnosis of wind turbine HSS gear spalling fault. This paper has shown that statistical CIs can provide global information about the condition of the gearbox at the same time being unreliable indicators of gear state condition. However, power in band CIs was able to provide specific information about the state of HSS gear. Results have shown the advantage of DWT filtering, using the TSA signal rather than using only the TSA signal, which improves the ability 
to diagnose earlier the fault signatures from the wind turbine gearbox. Also, results illustrate the following:

(i) Statistical condition indicators RMS, nRMS, CF, SKW, KURT, DA1, EO, FM0, ASB*, and NB4 based on the TSA signal do not reveal a linear increase over the wind turbine RDLT HSS gear fault progress.

(ii) Starting point of HSS gear fault is confirmed by SLF and SI as power in band CIs earlier than FM4 ${ }^{*}$ and M8A CIs based on difference signal.

(iii) P2P, SLF, SI, and NA4* extracted from approximation coefficient $A_{3}$ reveal fault progress better than extracted from regular TSA signal.

(iv) CIs that reveal useful information about HSS gear fault are more pertinent when extracted from the regular TSA and approximation coefficient $A_{3}$ (low frequency) signals than CIs extracted from detail coefficient $D_{3}$ (high frequency) signals.

(v) CIs ER, FM4, M6A, DiffDA1, and DiffP2P based on the difference signal do not reveal a linear increase over the wind turbine RDLT.

New approach presented in this paper has demonstrated the usefulness in wind turbine HSS gear spalling fault diagnosis and in tracking its progression over the time. The diagnosis results were confirmed with a wind turbine field case by gearbox site inspection.

\section{Conflict of Interests}

The authors declare that there is no conflict of interests regarding the publication of this paper.

\section{References}

[1] Global Wind Energy Council, Global Wind Statistics, 2014, http://www.gwec.net/wp-content/uploads/2015/02/GWEC_GlobalWindStats2014_FINAL_10.2.2015.pdf.

[2] BAN Research, International Wind Energy Development: World Market Update 2015, Navigant Consulting, Chicago, Ill, USA, 2013.

[3] P. J. Dempsey and S. Sheng, "Investigation of data fusion applied to health monitoring of wind turbine drivetrain components," Wind Energy, vol. 16, no. 4, pp. 479-489, 2013.

[4] I. Staffell and R. Green, "How does wind farm performance decline with age?” Renewable Energy, vol. 66, pp. 775-786, 2014.

[5] W. Yang, P. J. Tavner, C. J. Crabtree, Y. Feng, and Y. Qiu, "Wind turbine condition monitoring: technical and commercial challenges," Wind Energy, vol. 17, no. 5, pp. 673-693, 2014.

[6] Y. Feng, Y. Qiu, C. J. Crabtree, H. Long, and P. J. Tavner, "Monitoring wind turbine gearboxes," Wind Energy, vol. 16, no. 5, pp. 728-740, 2013.

[7] X. Wang, V. Makis, and M. Yang, "A wavelet approach to fault diagnosis of a gearbox under varying load conditions," Journal of Sound and Vibration, vol. 329, no. 9, pp. 1570-1585, 2010.

[8] N. Zuber, R. Bajrić, and R. Šostakov, "Gearbox faults identification using vibration signal analysis and artificial intelligence methods," Eksploatacja i Niezawodnosc, vol. 16, no. 1, pp. 61-65, 2014.
[9] Z. Feng, M. Liang, Y. Zhang, and S. Hou, "Fault diagnosis for wind turbine planetary gearboxes via demodulation analysis based on ensemble empirical mode decomposition and energy separation," Renewable Energy, vol. 47, pp. 112-126, 2012.

[10] P. Večeř, M. Kreidl, and R. Šmíd, "Condition indicators for gearbox condition monitoring systems," Acta Polytechnica, vol. 45, no. 6, pp. 35-43, 2005.

[11] Y. Lei, M. J. Zuo, Z. He, and Y. Zi, "A multidimensional hybrid intelligent method for gear fault diagnosis," Expert Systems with Applications, vol. 37, no. 2, pp. 1419-1430, 2010.

[12] J. Sheldon, G. Mott, H. Lee, and M. Watson, "Robust wind turbine gearbox fault detection," Wind Energy, vol. 17, no. 5, pp. 745-755, 2014.

[13] H. Luo, C. Hatch, M. Kalb, J. Hanna, A. Weiss, and S. Sheng, "Effective and accurate approaches for wind turbine gearbox condition monitoring," Wind Energy, vol. 17, no. 5, pp. 715-728, 2014.

[14] D. Siegel, W. Zhao, E. Lapira, M. AbuAli, and J. Lee, "A comparative study on vibration-based condition monitoring algorithms for wind turbine drive trains," Wind Energy, vol. 17, no. 5, pp. 695-714, 2014.

[15] Z. K. Peng and F. L. Chu, "Application of the wavelet transform in machine condition monitoring and fault diagnostics: a review with bibliography," Mechanical Systems and Signal Processing, vol. 18, no. 2, pp. 199-221, 2004.

[16] L. Hong and J. S. Dhupia, "A time domain approach to diagnose gearbox fault based on measured vibration signals," Journal of Sound and Vibration, vol. 333, no. 7, pp. 2164-2180, 2014.

[17] Y. Zhan, V. Makis, and A. K. S. Jardine, "Adaptive state detection of gearboxes under varying load conditions based on parametric modelling," Mechanical Systems and Signal Processing, vol. 20, no. 1, pp. 188-221, 2006.

[18] K. Smolders, H. Long, Y. Feng, and P. Tavner, "Reliability analysis and prediction of wind turbine gearboxes," in Proceedings of the European Wind Energy Conference and Exhibition (EWEC '10), pp. 162-165, Warsaw, Poland, April 2010.

[19] D. E. Newland, "Wavelet analysis of vibration: part 1-theory," Journal of Vibration and Acoustics, vol. 116, no. 4, pp. 409-416, 1994.

[20] R. Yan, R. X. Gao, and X. Chen, "Wavelets for fault diagnosis of rotary machines: a review with applications," Signal Processing. Part A, vol. 96, pp. 1-15, 2014.

[21] Z. Li, Z. Ma, Y. Liu, W. Teng, and R. Jiang, "Crack fault detection for a gearbox using discrete wavelet transform and an adaptive resonance theory neural network," Journal of Mechanical Engineering, vol. 61, no. 1, pp. 63-73, 2015.

[22] S. G. Mallat, "A theory for multiresolution signal decomposition: the wavelet representation," IEEE Transactions on Pattern Analysis and Machine Intelligence, vol. 11, no. 7, pp. 674-693, 1989.

[23] J. Rafiee, M. A. Rafiee, and P. W. Tse, "Application of mother wavelet functions for automatic gear and bearing fault diagnosis," Expert Systems with Applications, vol. 37, no. 6, pp. 45684579, 2010.

[24] G. Dalpiaz, A. Rivola, and R. Rubini, "Effectiveness and sensitivity of vibration processing techniques for local fault detection in gears," Mechanical Systems and Signal Processing, vol. 14, no. 3, pp. 387-412, 2000.

[25] F. P. García Márquez, A. M. Tobias, J. M. Pinar Pérez, and M. Papaelias, "Condition monitoring of wind turbines: techniques and methods," Renewable Energy, vol. 46, pp. 169-178, 2012. 
[26] P. J. Dempsey, D. R. Wade, L. J. Antolick, and J. Thomas, "Investigation of spiral bevel gear condition indicator validation via AC-29-2C using fielded rotorcraft HUMS data," Tech. Rep. NASA/TM-2014-218406, NASA, 2014.

[27] P. J. Rzeszucinski, J. K. Sinha, R. Edwards, A. Starr, and B. Allen, "Normalised root mean square and amplitude of sidebands of vibration response as tools for gearbox diagnosis," Strain, vol. 48, no. 6, pp. 445-452, 2012.

[28] L. J. Antolick, J. S. Branning, D. R. Wade, and P. J. Dempsey, "Evaluation of gear condition indicator performance on rotorcraft fleet," in Proceedings of the 66th Annual Forum of the American Helicopter Society, Phoenix, Ariz, USA, May 2010.

[29] P. D. Samuel and D. J. Pines, "A review of vibration-based techniques for helicopter transmission diagnostics," Journal of Sound and Vibration, vol. 282, no. 1-2, pp. 475-508, 2005.

[30] H. J. Decker, R. F. Handschuh, and J. J. Zakrajsek, "An enhancement to the NA4 gear vibration diagnostic parameter," Tech. Rep. ARL-TR-389, US Army Research Laboratory, Adelphi, Md, USA, 1994.

[31] H. Martin, "Statistical moment analysis as a means of surface damage detection," in Proceedings of the 7th International Modal Analysis Conference, pp. 1016-1021, Las Vegas, Nev, USA, February 1989. 


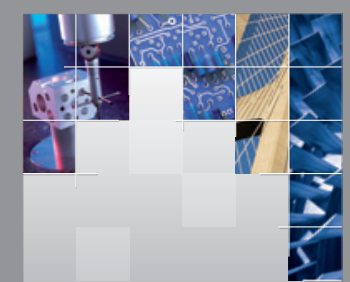

\section{Enfincering}
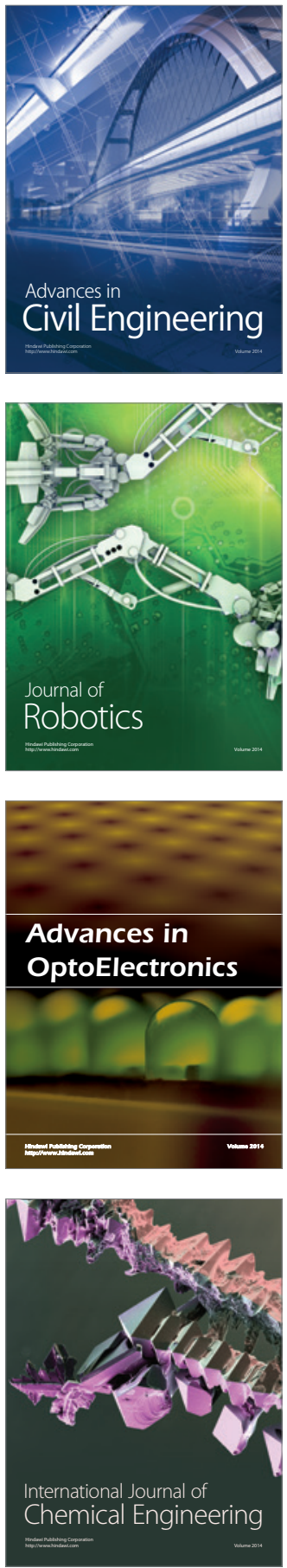

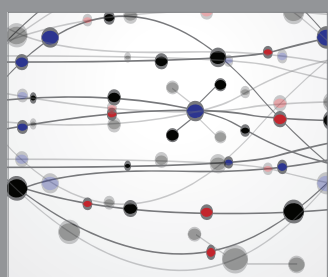

The Scientific World Journal

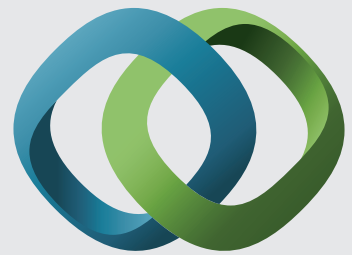

\section{Hindawi}

Submit your manuscripts at

http://www.hindawi.com
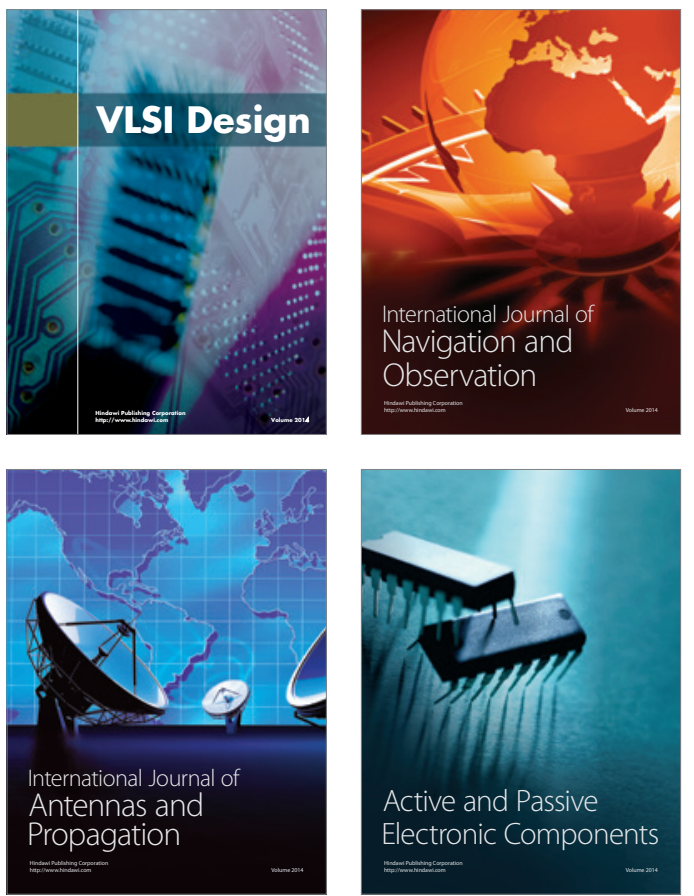
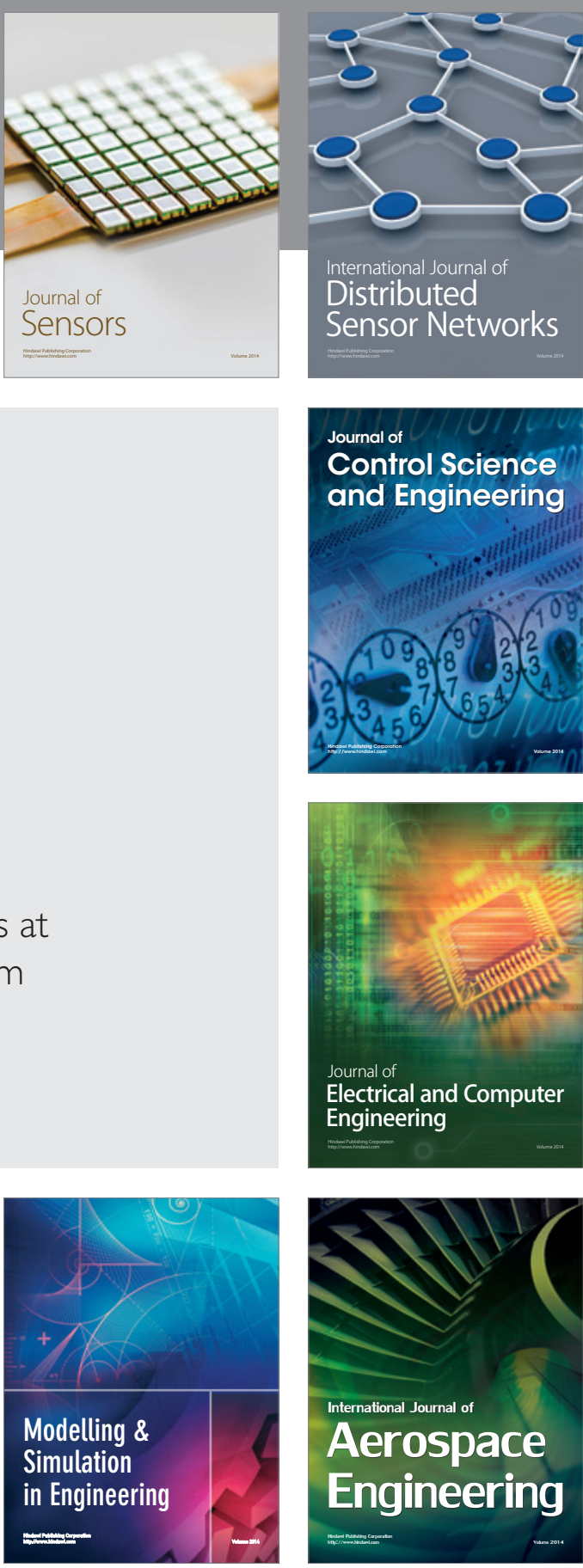

International Journal of

Distributed

Sensor Networks

Journal of

Control Science

and Engineering
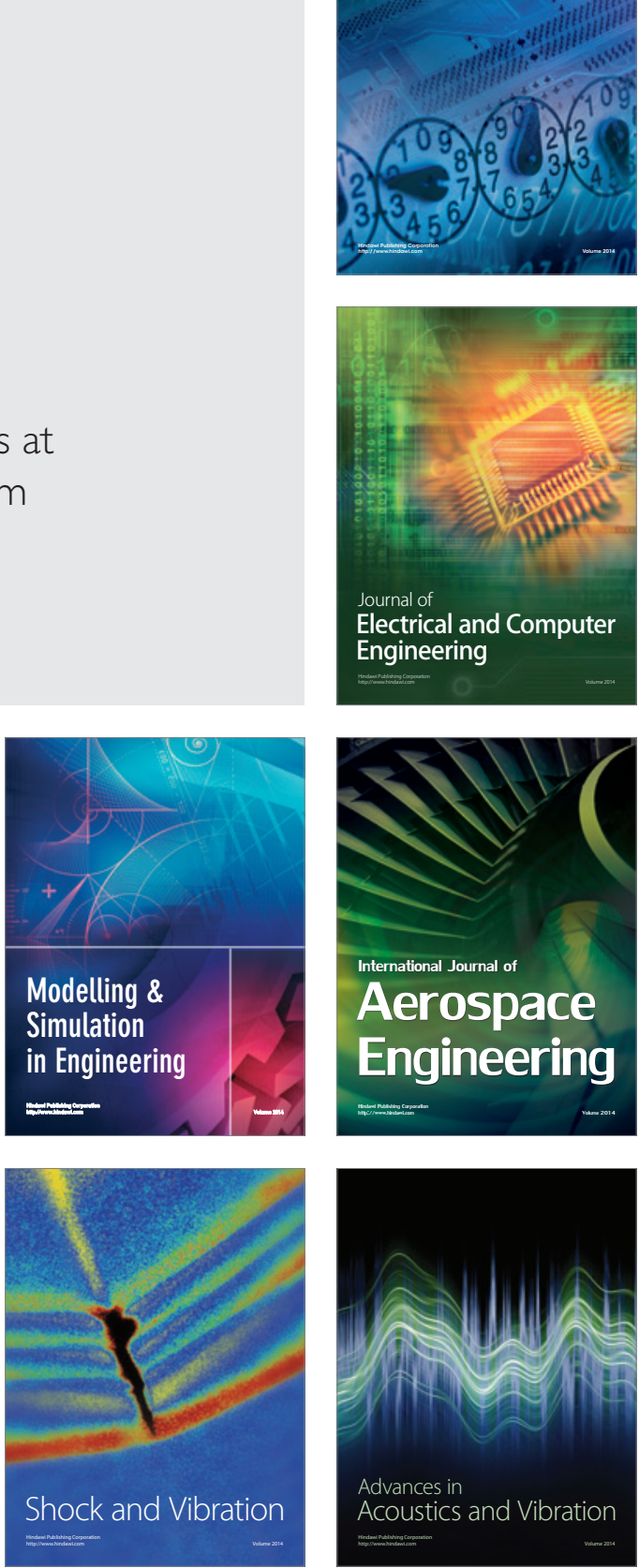